\title{
Spectral ageing in the relic radio galaxy B2 0924+30
}

\author{
M. Jamrozy ${ }^{1}$, U. Klein ${ }^{1}$, K.-H. Mack ${ }^{1,2,3}$, L. Gregorini ${ }^{2,4}$, and P. Parma ${ }^{2}$ \\ 1 Radioastronomisches Institut der Universität Bonn, Auf dem Hügel 71, 53121 Bonn, Germany \\ e-mail: mjamrozy@astro.uni-bonn.de \\ 2 Istituto di Radioastronomia, via P. Gobetti 101, 40129 Bologna, Italy \\ 3 ASTRON, Postbus 2, 7990 AA Dwingeloo, The Netherlands \\ ${ }^{4}$ Dipartimento di Fisica, Università di Bologna, via Irnerio 46, 40126 Bologna, Italy
}

Received 7 April 2004 / Accepted 8 July 2004

\begin{abstract}
The radio spectrum of the relic radio galaxy B2 0924+30 is investigated using integrated flux densities between 0.151 and $10.6 \mathrm{GHz}$, as well as images at $0.325,0.609,1.400$ and $4.750 \mathrm{GHz}$. Allowing for synchrotron and inverse Compton losses in this source, the observed spectral steepening yields a break frequency of $\sim 7 \mathrm{GHz}$, implying a mean particle age of about 50 Myrs. The corresponding particle advance speed is of the order of $2000 \mathrm{~km} \mathrm{~s}^{-1}$. This relatively small particle lifetime following the exhaustion of the central source can explain the paucity of such sources and implies that such objects would easily escape detection if searched for in the $\mathrm{cm}$ wavelength regime.
\end{abstract}

Key words. radio continuum: galaxies - galaxies: active - galaxies: individual: B2 0924+30

\section{Introduction}

Aged radio galaxies have seen increasing interest in recent years because their investigation has become feasible at high radio frequencies due to the improvements of sensitivity in radio astronomical measurements. To avoid misunderstandings and confusion in commonly used names of extended lowbrightness and steep-spectrum radio sources associated with clusters of galaxies we follow the taxonomy proposed by Kempner et al. (2003) who reserve the name radio relics for sources associated with extinct or dying active galactic nuclei (AGN) only. These relic sources are important for the understanding of radio source evolution, in particular the late phase of exhaustion of the central energy sources, the AGN. Their relative paucity is still puzzling: on the one hand, there are numerous bright elliptical galaxies that lack radio counterparts, while there are certainly as many bright ellipticals that do possess AGN, in some form of the so-called "extragalactic zoo", often along with the classical double-lobe structure associated with radio galaxies. In the light of this, the scarceness of relic sources must imply that both the "switch-off" of the central energy source as well as the fading of the radio lobes takes place relatively rapidly. On the other hand, the few existing candidate sources provide the (almost) unique opportunity of estimating at least the duration of the decline of the lobe brightness, owing to the pronounced spectral steepening by which they are characterised. Another essential condition is the absence of any central source, and of any coherent jet structure, both of which would hint at ongoing activity and transport of energy and momentum out into the lobes.
The evolution of the synchrotron spectra of diffuse radio sources has been the subject of a number of papers (Schlickeiser et al. 1987; Komissarov \& Gubanov 1994; Goldshmidt \& Raphaeli 1994; Kaiser \& Cotter 2002). The pronounced steepening seen in the integrated radio spectra of relics, and in particular in their spectral index distributions, makes them ideal cases to discuss different energy loss schemes, viz. synchrotron losses with (Jaffe \& Perola 1973) or without (Kardashev 1962; Pacholczyk 1970) re-isotropisation of pitch angles, and inverse Compton losses. These calculations have so far mainly been carried out for diffuse radio continuum structures of galaxy clusters.

The sources B0917+75 (Harris et al. 1993), B2 0924+30 (this paper), J1324-3138 (Venturi et al. 1998) and three other objects found by Parma et al. (2003) are examples of dying radio galaxies.

The large double radio source B2 0924+30 may be considered a prototypical genuine relic of a "dead" radio galaxy as it seems to fulfill perfectly the mentioned above criteria: its overall radio spectrum is rather steep, with the spectral index in the range $\alpha=-1.0$ to $-1.2 \pm 0.1$ already at low radio frequencies (Ekers et al. 1981; Cordey 1987). Its core luminosity is by far the lowest known so far: the upper limit set by the $5-\mathrm{GHz}$ measurements of Giovannini et al. (1988) is up to 5 orders of magnitude below the strongest known core sources, and still falls about two orders of magnitude below the value expected from the correlation of total and core luminosities of elliptical galaxies as e.g. derived by Giovannini et al. (1988).

B2 0924+30 is associated with the bright $\left(m_{\mathrm{pg}} \approx 14 \mathrm{~m} 5\right.$, Nilson 1973) E/S0 type galaxy IC 2476 (UGC 05043) which is 
the central object in a group of 8 galaxies (Mahdavi et al. 1999; White et al. 1999). The low resolution optical spectrum of the galaxy $^{1}$ (Falco et al. 1999) does not show any distinct emission lines and looks like a typical spectrum of a non-active galaxy. The ROSAT data towards B2 0924+30 suggest very weak extended X-ray emission which may be connected with intergalactic hot gas (Canosa et al. 1999).

The first radio continuum detection of this relic radio galaxy was made at $0.408 \mathrm{GHz}$ (Colla et al 1970, 1975) and later its structure was mapped by Ekers et al. $(1975,1981)$ at $0.6,1.4$ and $5 \mathrm{GHz}$. This investigation was followed by lowfrequency observations at $0.151 \mathrm{GHz}$ (Cordey 1987) and high frequency single-dish measurements at $10.55 \mathrm{GHz}$ (Gregorini et al. 1992). The radio continuum morphology of B2 0924+30 suggests that the source might be a remnant of an edgebrightened FRII (Fanaroff \& Riley 1974) type galaxy. The relic does not show any distinct hot spots, although the regions with the flattest spectral index in the lobes may be a place where the past hot spots were located. The $1.4 \mathrm{GHz}$ luminosity of the source is about $10^{23.8} \mathrm{~W} \mathrm{~Hz}^{-1}$ which is less than those of a typical high-luminosity radio galaxy. It is likely, however, that the progenitor of the relic was more powerful but after the "switchoff" of its central activity diminished its luminosity mostly due to diffusion of the relativistic electrons as well as through synchrotron and inverse Compton emission.

In this paper, we report radio continuum observations of B2 0924+30 which allow a study of the spectral index distribution across the source over a wide frequency range. The observed spectral steepening will be utilised to establish a precise source age since "switch-off".

In Sect. 2 we describe the observations and data analysis. In Sect. 3 the results are presented. In Sect. 4 we derive the total radio spectrum and the distribution of the spectral index across the source. The spectrum is discussed in terms of models of particle energy losses, and the source age since "switch-off" is estimated. Our results and conclusions are summarised in Sect. 5 .

Throughout this paper we will assume $q_{0}=0.5$ and a Hubble constant of $H_{0}=71 \mathrm{~km} \mathrm{~s}^{-1} \mathrm{Mpc}^{-1}$, which, with the redshift of 0.02692 (Falco et al. 1999), places B2 0924+30 at a comoving radial distance of about $110 \mathrm{Mpc}$. The spectral in$\operatorname{dex} \alpha$ is defined here according to the relation $S_{\nu} \propto v^{\alpha}$ between flux density $S_{v}$ and frequency $v$.

\section{Observations and data reduction}

In order to perform a spectral ageing analysis of B2 0924+30 we used images at $\lambda \lambda 2.8$ (from Gregorini et al. 1992) and $6.3 \mathrm{~cm}$ (observed with the Effelsberg 100-m telescope), $21 \mathrm{~cm}$ (from the NRAO VLA Sky Survey, NVSS, Condon et al. 1998), $49 \mathrm{~cm}$ (dedicated observations with the Westerbork Synthesis Radio Telescope, WSRT), and at $92 \mathrm{~cm}$ (from the Westerbork Northern Sky Survey, WENSS, Rengelink et al. 1997). Because of the lack of short baselines, the NVSS is insensitive to extended structures. Hence, the total flux-density of B2 0924+30

\footnotetext{
1 The spectrum is available in electronic form at http://tdc-www.harvard.edu/uzc/
}

measured in the NVSS image is $(312.7 \pm 10.4) \mathrm{mJy}$. This value is only about $75 \%$ of the value derived from the radio spectrum of the entire source. In order to include missing short-spacing data we combined the NVSS image with a single-dish observation carried out with the Effelsberg 100-m telescope. The common flux density scale of our measurements is that of Baars et al. (1977).

\subsection{The $\lambda 6.3-\mathrm{cm}$ data}

The observations at $\lambda 6.3 \mathrm{~cm}$ were carried out between June and November 1994, using the twin-beam receiver system in the secondary focus of the Effelsberg 100-m telescope. The receiver system was that described e.g. by Gioia et al. (1982), correlating one circular polarisation from the main and the offset horn, which are 7'.96 apart on the sky, to yield the power difference (Stokes $I$ ) of the two feeds. The two circular polarisations of the main horn were turned into Stokes parameters $U$ and $Q$ by means of an IF polarimeter. The differential total power image was restored to the equivalent single-beam image using the algorithm of Emerson et al. (1979). In this way, a total of 126 coverages were obtained in order to detect the faint emission from this steep-spectrum object. Each coverage was $28^{\prime} \times 20^{\prime}$ wide in azimuth and elevation, with a scanning speed of $40^{\prime} / \mathrm{min}$ and $1^{\prime}$ scan separation. The final image, centred on $\alpha_{\mathrm{J} 2000}=09^{\mathrm{h}} 27^{\mathrm{m}} 47^{\mathrm{s}} .4, \delta_{\mathrm{J} 2000}=29^{\circ} 58^{\prime} 17^{\prime \prime} .9$, was created by a weighted average (weights proportional to the inverse squares of the rms noise levels) of the individual coverage, after retabulation onto a right ascension declination grid. Its rms noise is 1.53 and $1.05 \mathrm{mJy} / \mathrm{beam}$ area in total power and polarised intensity, respectively. The half-power beam width was determined to be $147^{\prime \prime}$. The telescope pointing was frequently checked by cross-scanning the sources 4C 39.25, 3C 123, $3 \mathrm{C} 138$, and $3 \mathrm{C} 286$, with the latter three also serving as flux and polarisation calibrators via mapping.

\subsection{The $\lambda 21-\mathrm{cm}$ data}

The single-dish $\lambda 21-\mathrm{cm}$ measurements were carried out with the Effelsberg 100-m telescope in September 2003, using the 1-horn, 2-channel receiver installed in the primary focus of the telescope. The final image was a combination of individual images scanned alternately in right ascension and declination. In this way, a total of 6 coverages were obtained. The images were large enough to cover the source plus some emission-free areas used for determination of zero levels and noise. The drive rate was $180^{\prime} / \mathrm{min}$ and the scan interval $3^{\prime}$. The combination was made using the "basket-weaving" technique (Sieber et al. 1979). The image centre is $\alpha_{\mathrm{J} 2000}=09^{\mathrm{h}} 27^{\mathrm{m}} 49^{\mathrm{s}} .8$, $\delta_{\mathrm{J} 2000}=29^{\circ} 59^{\prime} 19^{\prime}{ }^{\prime} 8$. The flux density and polarisation calibration was performed using the standard calibration sources 3C 286 and 3C 138.

Combination of the single-dish and NVSS images has been performed for Stokes $I, Q$ and $U$ using IMERG included in the AIPS package, which permits to merge two input images in the $(u, v)$ plane. The final rms noise of the image is 0.56 
Table 1. Flux densities for Source B.

\begin{tabular}{ccc}
\hline \hline$v[\mathrm{GHz}]$ & $S_{v}[\mathrm{mJy}]$ & Reference \\
\hline 0.151 & $150 \pm 22$ & Cordey (1987) \\
0.325 & $101 \pm 6$ & This work (WENSS) \\
0.609 & 80 & Ekers et al. (1981) \\
0.609 & $73 \pm 2$ & This work \\
1.400 & $59 \pm 1$ & This work (NVSS) \\
1.400 & 54 & Ekers et al. (1981) \\
4.750 & $53 \pm 2$ & This work \\
5.000 & 32 & Ekers et al. (1981) \\
10.55 & $22 \pm 2$ & Gregorini et al. (1992) \\
\hline
\end{tabular}

and $0.3 \mathrm{mJy} / \mathrm{beam}$ area in total power and polarised intensity, respectively.

\subsection{The $\lambda 49-\mathrm{cm}$ data}

The $\lambda 49-\mathrm{cm}$ measurements were made with the WSRT in July 1985 , with a full $12 \mathrm{~h}$ synthesis spent on B2 $0924+30$. The field centre is $\alpha_{\mathrm{J} 2000}=09^{\mathrm{h}} 27^{\mathrm{m}} 52^{\mathrm{s}} .9, \delta_{\mathrm{J} 2000}=29^{\circ} 59^{\prime} 09^{\prime} \cdot 7$. The data have been Fourier transformed and CLEANed in a standard way with the AIPS package. The final rms noise of the image is $0.77 \mathrm{mJy} /$ beam area in the full resolution image (beam size $\left.29^{\prime \prime} \times 56^{\prime \prime}\right)$.

\subsection{The $192-\mathrm{cm}$ data}

In order to obtain a low-frequency data set of B2 0924+30 we have used the publicly available database of the WENSS. This radio continuum survey at $325 \mathrm{MHz}$ covers the whole sky north of $\delta \sim 30^{\circ}$ to a limiting flux density of unresolved sources of about $18 \mathrm{mJy}$ (at the $5 \sigma$ level).

The resolution of the image is about $54^{\prime} \times 108^{\prime}$ and the rms noise across this field is $3.6 \mathrm{mJy} / \mathrm{beam}$ area.

\section{Results}

\subsection{Radio images}

Figures 1 through 4 show the total power images of B2 0924+30 at $\lambda \lambda 6.3,21,49$ and $92 \mathrm{~cm}$. In Figs. 1a and 1b we present the $\lambda 6.3-\mathrm{cm}$ image. The bright source located at $\alpha_{\mathrm{J} 2000}=09^{\mathrm{h}} 27^{\mathrm{m}} 22^{\mathrm{s}} .8, \delta_{\mathrm{J} 2000}=29^{\circ} 54^{\prime} 14^{\prime \prime} \cdot 6$ (Fig. 1a, designated Source B by Ekers et al. 1975) is unrelated to B2 0924+30 and has been subtracted from the image (Fig. 1b). We have compiled the flux densities of this source, which was identified with a quasar (Ekers et al. 1981; Arp 1977), in Table 1. Except for the 4750-MHz flux density, its spectrum obeys a perfect powerlaw, with spectral index $\alpha_{151}^{10550}=-0.44 \pm 0.02$. However, the $\lambda 6.3-\mathrm{cm}$ value is $\sim 60 \%$ higher than what is inferred from the overall spectrum, implying that this source may exhibit some kind of variability.

In spite of the relatively low angular resolution of our 16.3-cm image a few features can be seen in Fig. 1b. The two lobes are clearly discernible. On the southern side, both
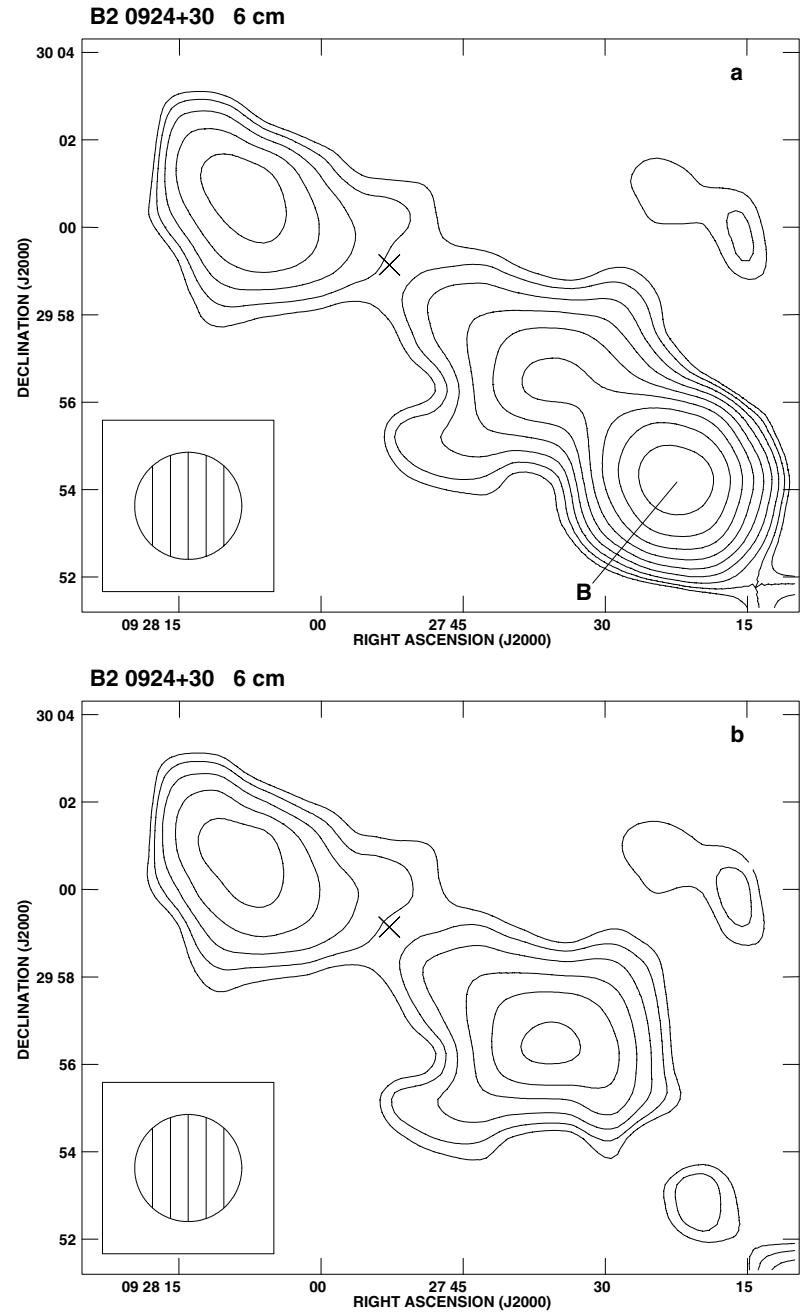

Fig. 1. Image of the total intensity of $\mathrm{B} 20924+30$ at $\lambda 6.3 \mathrm{~cm}$. The cross indicates the position of the host galaxy IC 2476. Contours are: $1, \sqrt{2}, 2, \sqrt{2}^{3}, \ldots \times 2.5 \mathrm{mJy} / \mathrm{beam}$ area. The hatched circle in the lower left mimics the half-power beam size. a) The bright unrelated source (marked with the letter B) is still present in this image. b) The confusion source has been subtracted in this image.

of them exhibit faint extensions, while in the north the outer contour depicts a rather straight edge. This behaviour is also seen in the low-frequency images of Ekers et al. (1981) and Cordey (1987). Linear polarisation is only marginally detected at $\lambda 6.3 \mathrm{~cm}$ (not shown here). If real, degrees of polarisation of up to $\sim 30 \%$ are indicated in the lobes.

In order to illustrate the differences between the pure NVSS and the combined image we have drawn contours of equal levels in both Figs. 2 and 5a, respectively. The combined 121-cm image (Fig. 2) reveals two prominent lobes connected by a continuous bridge of synchrotron emission. The original NVSS image (contours in Fig. 5a) of B2 0924+30 shows two detached radio lobes. An extended source is visible in the centre. In order to find evidence of any nuclear activity of B2 0924+30 we check the high resolution $\left(5^{\prime \prime} \times 5^{\prime \prime}\right)$ image of the source from the 1.4-GHz FIRST survey (Becker et al. 1995). The inspection shows that there are no compact sources 


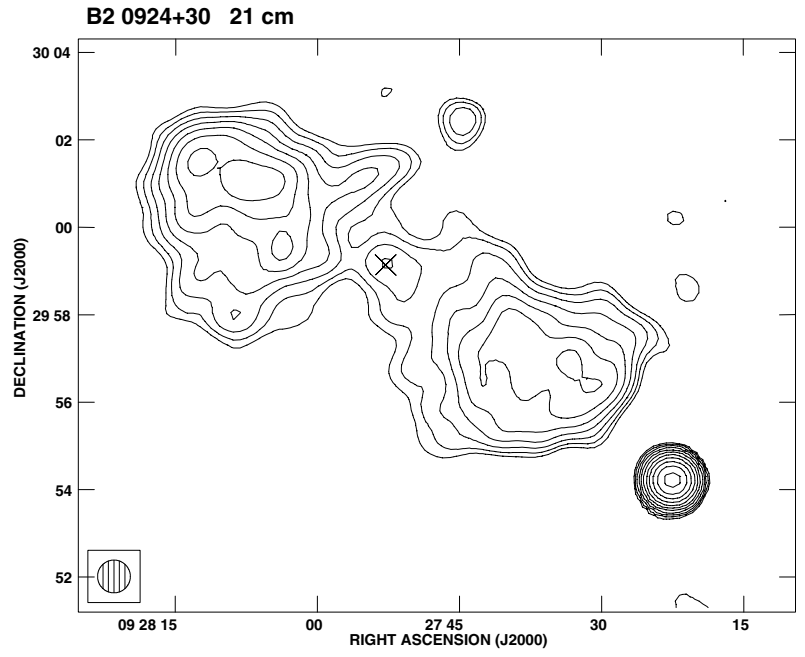

Fig. 2. Image of the total intensity of B2 $0924+30$ at $\lambda 21 \mathrm{~cm}$. Contours are: $1, \sqrt{2}, 2, \sqrt{2}^{3}, \ldots \times 1.5 \mathrm{mJy} / \mathrm{beam}$ area. Otherwise the layout is the same as in Fig. 1.

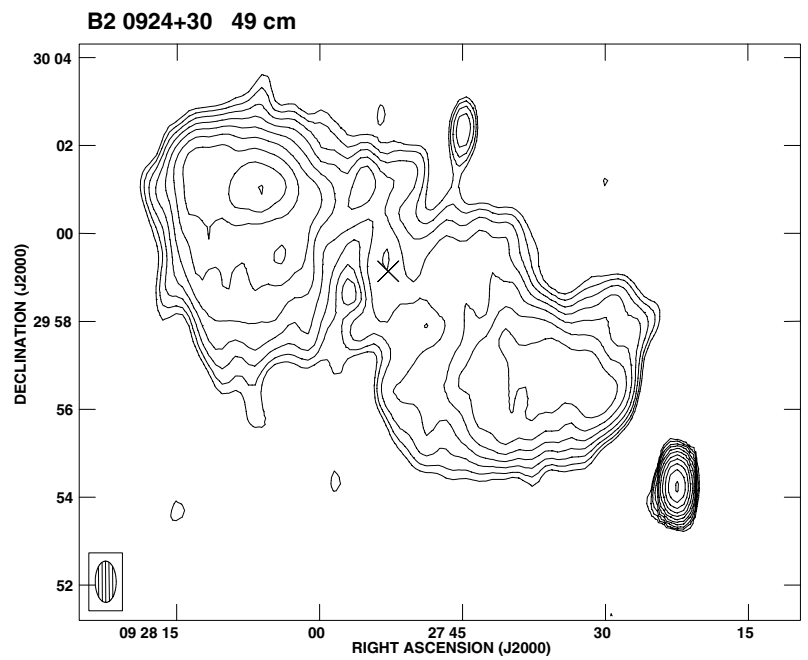

Fig. 3. Image of the total intensity of B $20924+30$ at $\lambda 49 \mathrm{~cm}$. Contours are: $1, \sqrt{2}, 2, \sqrt{2}^{3}, \ldots \times 2.0 \mathrm{mJy} / \mathrm{beam}$ area. Otherwise the layout is the same as in Fig. 1 .

within a $2^{\prime}$ diameter circular field centred on the position of the host galaxy down to the rms noise level of $0.14 \mathrm{mJy} /$ beam.

In Fig. 3 we present the $149-\mathrm{cm}$ image of B2 0924+30 at full resolution. The mentioned above features are evident in this image as well.

For completeness we also show the $192-\mathrm{cm}$ image (Fig. 4) of B2 $0924+30$. The position of the radio core is not evident in this image at all.

We have smoothed the $\lambda \lambda 21,49$ and $92-\mathrm{cm}$ total-power images to the $147^{\prime \prime}$ beam size of the $\lambda 6.3-\mathrm{cm}$ Effelsberg image in order to carry out the spectral (Sect. 3.2) and age analysis (Sect. 4).

The linear polarisation images of the relic at $\lambda 21 \mathrm{~cm}$ are also shown in Fig. 5. At this frequency, the electric field E-vectors have a regular pattern which suggests that the largescale magnetic field in the source is well organised. Significant polarisation is seen, the degrees reaching $\sim(3.9 \pm 0.7) \%$. The

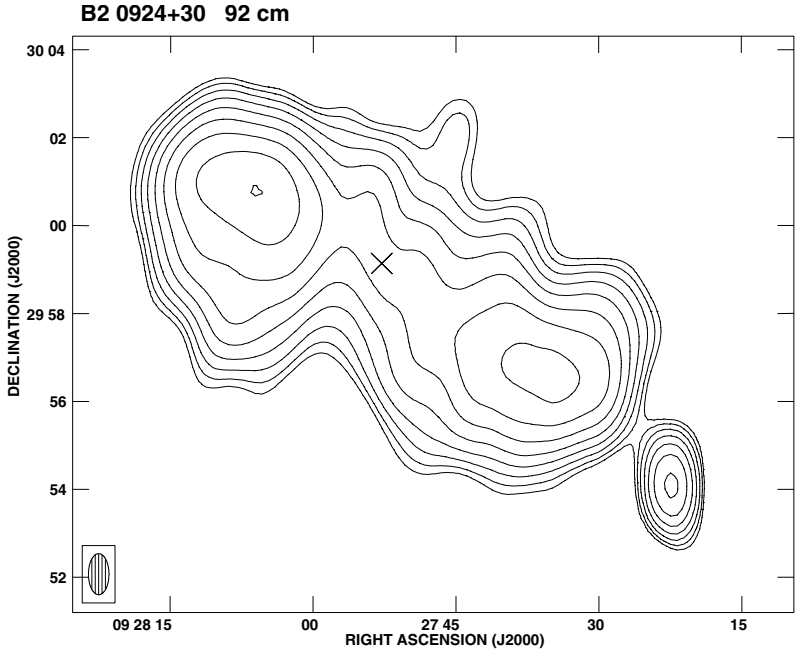

Fig. 4. Image of the total intensity of B2 0924+30 at $\lambda 92 \mathrm{~cm}$. Contours are: $1, \sqrt{2}, 2, \sqrt{2}^{3}, \ldots \times 11 \mathrm{mJy} /$ beam area. Otherwise the layout is the same as in Fig. 1.

Table 2. Flux densities for B2 0924+30.

\begin{tabular}{ccc}
\hline \hline$v[\mathrm{GHz}]$ & $S_{v}[\mathrm{mJy}]$ & Reference \\
\hline 0.151 & $4600 \pm 360$ & Cordey (1987) \\
0.325 & $2425 \pm 124$ & This work (WENSS) \\
0.408 & 1860 & Colla et al. (1975) \\
0.609 & $1094 \pm 56$ & This work \\
0.609 & 1060 & Ekers et al. (1981) \\
1.400 & $420 \pm 43$ & This work (NVSS) \\
1.400 & 442 & Colla et al. (1975) \\
4.750 & $60 \pm 7$ & This work \\
10.55 & $10 \pm 4$ & Gregorini et al. (1992) \\
\hline
\end{tabular}

polarised intensity seems to mark the edges of the lobes in a nearly "S-shaped" morphology. In order to determine the distribution of the magnetic field directions it is necessary to correct the $\lambda 21-\mathrm{cm}$ data for the Faraday rotation measure gradients and for that purpose subsequent radio polarisation measurements at other wavelengths are needed.

\subsection{Spectral index}

We have computed the total flux densities of B2 0924+30 by integrating the four images (Figs. 1-4) within a polygon adapted to the overall source shape, with careful inspection of the image zero-levels, and Source B subtracted. These flux densities are collected in Table 2, along with flux densities published in the literature. The spectrum is plotted in Fig. 6. A pronounced steepening is seen beyond about $2 \mathrm{GHz}$. Between 151 and $408 \mathrm{MHz}$ the spectral index is $\alpha_{151}^{408}=-0.89 \pm 0.06$, while between 4750 and $10550 \mathrm{MHz}$ it is $\alpha_{4750}^{10550}=-2.25 \pm 0.52$, implying a steepening by $|\Delta \alpha|=1.4$, which is significant in spite of the relatively large error at the highest frequency.

We have used the images of $147^{\prime \prime}$ resolution each in order to compute the spectral index across B2 0924+30 between $\lambda \lambda 21$ and $6.3 \mathrm{~cm}, 49$ and $21 \mathrm{~cm}$, and 92 and $49 \mathrm{~cm}$. The results are 

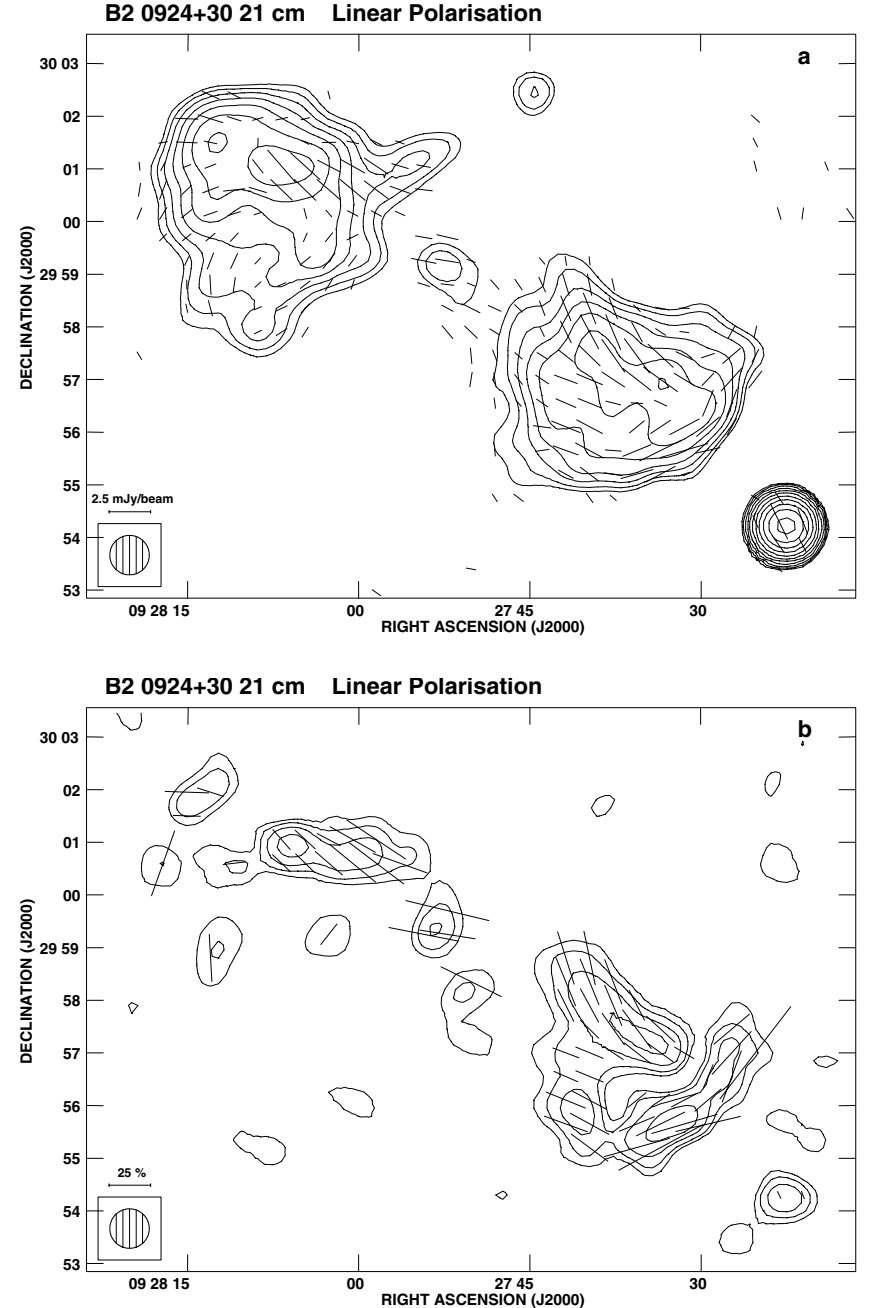

Fig. 5. Images of the linear polarisation of B2 0924+30 at $\lambda 21 \mathrm{~cm}$. a) Electric field E-vectors, with their length proportional to the polarised intensity, superimposed with $1, \sqrt{2}, 2, \sqrt{2}^{3}, \ldots \times 1.5 \mathrm{mJy} / \mathrm{beam}$ total intensity contours from the NVSS. b) Linearly polarised intensity contours of $1, \sqrt{2}, 2, \sqrt{2}^{3} \times 0.9 \mathrm{mJy} / \mathrm{beam}$, with the vectors of the fractional linear polarisation superimposed. The scales of the linear polarisation vectors are indicated by the bars and the half-power beam size by the circles in the lower left corners of the images.

shown in Fig. 7. The spectrum strongly steepens, both from the outer regions towards the centre of the source and outwards perpendicular to the major axis of the objects, similar to what is seen in classical FRII and some double lobed FRI (Parma et al. 1999) radio sources whose lobes are filled with backflow material which is older in the centre than on the edges of the radio galaxy.

The high-frequency spectral index image derived between $\lambda \lambda 6.3$ and $21 \mathrm{~cm}$ shows values of $\alpha_{1400}^{4750}=-1.4 \pm 0.1$, with a further steepening to $\alpha_{1400}^{4750}=-1.7 \pm 0.4$ around the host galaxy IC 2476 (marked by the cross). This general behaviour is qualitatively the same as that reported by Ekers et al. (1981). No significant asymmetry of the spectrum of the lobes is evident. The apparent flattening seen towards the edge of the western lobe may be related to the influence of the residuals of Source B discussed in Sect. 3.1.

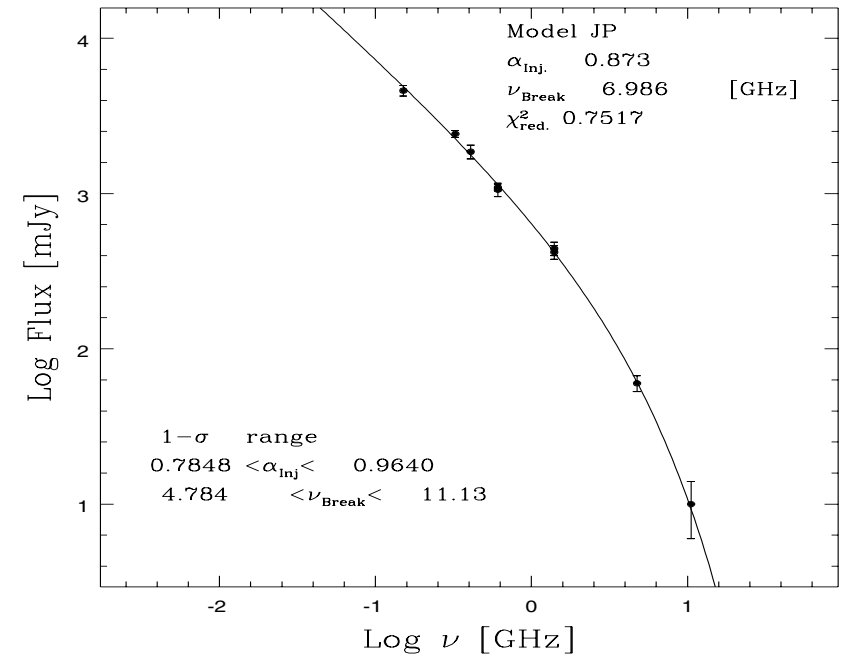

Fig. 6. Integral radio spectrum of B20924+30 between 151 and $10550 \mathrm{MHz}$. The solid line is a fit to the data (for details see Sect. 4).

An even more pronounced steepening of the spectrum is seen away from the major axis, where the spectrum steepens to $\alpha_{1400}^{4750}=-1.7 \pm 0.2$. This is reminiscent of the source B2 1321+31 (Klein et al. 1995), although with a less pronounced steepening in that case. The steepening in B2 0924+30 would be even more dramatic if followed up to $10.55 \mathrm{GHz}$, but unfortunately the image obtained by Gregorini et al. (1992) at this frequency lacks the sensitivity to allow a useful spectral comparison. Smoothing this image to the 147" beam size, spectral indices of $\alpha_{4750}^{10550}=-2.2 \pm 0.3$ in the eastern lobe and $\alpha_{4750}^{10550}=-4 \pm 1$ in the western one are indicated, corroborating the trend visible in the integral spectrum of Fig. 6.

A similar tendency in the spectral index distribution is seen at low frequencies, the average spectrum being flatter in this case. A more quantitative analysis will be presented in Sect. 4 .

\section{Discussion}

Both the integrated spectrum of B2 0924+30 (Fig. 6) as well as its spectral index distribution (Fig. 7) have been analysed in order to determine the characteristic break frequency of the synchrotron emission and corresponding particle ages, assuming that synchrotron and inverse Compton losses are at work.

For an obviously aged source like B2 0924+30, expansion losses can be ruled out. The lack of any central source and hot spots suggests that particle acceleration is no longer taking place. Therefore, a model describing continuous injection (which would refer to the source as a whole) can be excluded. Such a model would predict a steepening of the initial injection spectrum $\left(\alpha_{i}\right)$ by $|\Delta \alpha|=0.5$. Injection spectra observed in the hot spots of radio galaxies suggest $\alpha_{i} \approx-0.5$ (see e.g. Komissarov \& Gubanov 1994). However, even in the lowfrequency regime the spectral indices are lower than $\alpha=-1$ anywhere in the source so that ongoing injection must be ruled out.

The model described by Kardashev (1962) and Pacholczyk (1970), hereafter referred to as the KP model, assumes that no pitch angle scattering of the relativistic particle occurs, and 

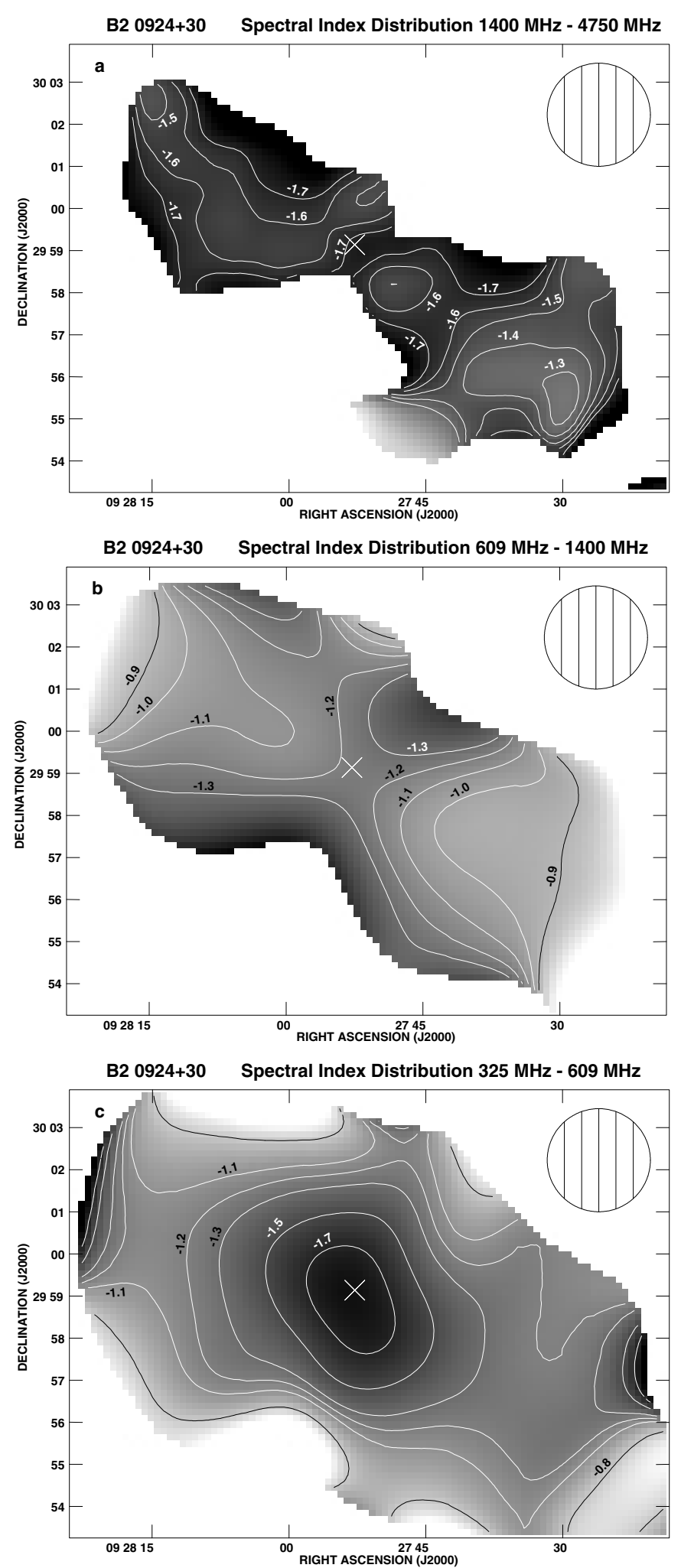

Fig. 7. Distribution of the spectral index of B2 0924+30. a) Between $\lambda \lambda 21$ and $6.3 \mathrm{~cm}$, b) between $\lambda \lambda 49$ and $21 \mathrm{~cm}$ and c) between $\lambda \lambda 92$ and $49 \mathrm{~cm}$. Spectral index contours are superimposed onto the spectral index grey scale.

predicts a high-frequency tail in the synchrotron spectrum, with a steepening to $\alpha=\left(4 / 3 \cdot \alpha_{i}\right)-1$ in the absence of inverse Compton losses. While the latter condition may be applicable to bright sources like Cyg A, in which the energy density of the lobe magnetic field dominates by far that of the microwave background (Carilli et al. 1991), this is certainly not true for relic sources. In this case, even non-isotropised particles produce an exponential high-frequency cut-off.

We therefore favour the model of Jaffe \& Perola (1973; hereafter called JP model), which allows for permanent pitch angle isotropisation by Alfvén waves created by the streaming motions of the particles in the irregular magnetic field. This model, considering both, synchrotron as well as inverse Compton losses, should then lead to an exponential cut-off in the radio spectrum.

The spectrum of the integrated radio emission of B2 0924+30 comprises reliable measurements at 7 frequencies so that a meaningful fit to the data should be possible. In addition, we have images at four frequencies at our disposal as well. Using the SYNAGE package (Murgia 1996) we have performed a fit to the entire source spectrum and computed the break frequency.

To derive the synchrotron age of the relic the strength of the magnetic field is required. We infer it from the usual equipartition arguments. We use the following equation of Miley (1980):

$B_{\text {eq }}=7.91 \cdot\left[\frac{(1+k)}{(1+z)^{\alpha-3}} \cdot \frac{S_{\mathrm{o}}}{v_{\mathrm{o}}^{\alpha} \theta_{x} \theta_{y} s} \cdot \frac{v_{u}^{\alpha+\frac{1}{2}}-v_{l}^{\alpha+\frac{1}{2}}}{\alpha+\frac{1}{2}}\right]^{\frac{2}{7}} \mu \mathrm{G}$.

Here, $S_{0}$ is the flux density (in mJy) at the observing frequency $v_{\mathrm{o}}$ (in $\mathrm{GHz}$ ), $k$ is the ratio of the energy content in relativistic protons to that of electrons (adopted as $k=1$ ), $v_{l}$ and $v_{u}$ are the integration boundaries for the radio luminosity $\left(v_{l}=0.01 \mathrm{GHz}\right.$ and $v_{u}=100 \mathrm{GHz}$ used here), $s$ is the path length through the source in the line of sight (in kpc), and $\theta_{x}$ and $\theta_{y}$ are source extents in two directions, measured in arcsec. For B2 0924+30 we have adopted a source extent of $4^{\prime}$ which corresponds to $\sim 130 \mathrm{kpc}$. This value is also used for the path length. The spectral index is the mean value derived from the four maps, which is $\approx-1.4$. The resulting field strength is $B_{\text {eq }} \approx 1.6 \mu \mathrm{G}$.

Using the values compiled in Table 2, we have applied a least-squares fit to the flux densities, which is also shown graphically in Fig. 6. The best fit $\left(\chi^{2}=0.75\right)$ to the JP model was obtained for a break frequency $v_{\mathrm{b}}=7.0_{-2.2}^{+4.1} \mathrm{GHz}$ and an "injection" spectral index of $\alpha_{\mathrm{i}}=-0.87 \pm 0.09$. Using the equation given by Alexander \& Leahy (1987) and the known equipartition magnetic field strength, as well as the break frequency resulting from the fit to the radio spectrum, we can calculate the particle ages $\tau$ since switch-off:

$\tau=1.59 \times 10^{3} \cdot v_{\mathrm{b}}^{-0.5} \cdot \frac{B_{\mathrm{eq}}^{0.5}}{B_{\mathrm{eq}}^{2}+B_{\mathrm{m}}^{2}} \mathrm{Myr}$.

Here, $B_{\text {eq }}$ is the equipartition field (in $\mu \mathrm{G}$ ), $B_{\mathrm{m}}$ the equivalent field of the $2.7-\mathrm{K}$ background radiation $(3.4 \mu \mathrm{G}$ at this redshift), and $v_{\mathrm{b}}$ the break frequency (in $\mathrm{GHz}$ ). From the above we obtain a particle age of $54_{-11}^{+12}$ Myrs, which must be considered as the average age of the particles in this object. The "injection" spectral index does naturally not refer to hot spots which are still energised by the relativistic plasma beams. The former hot 


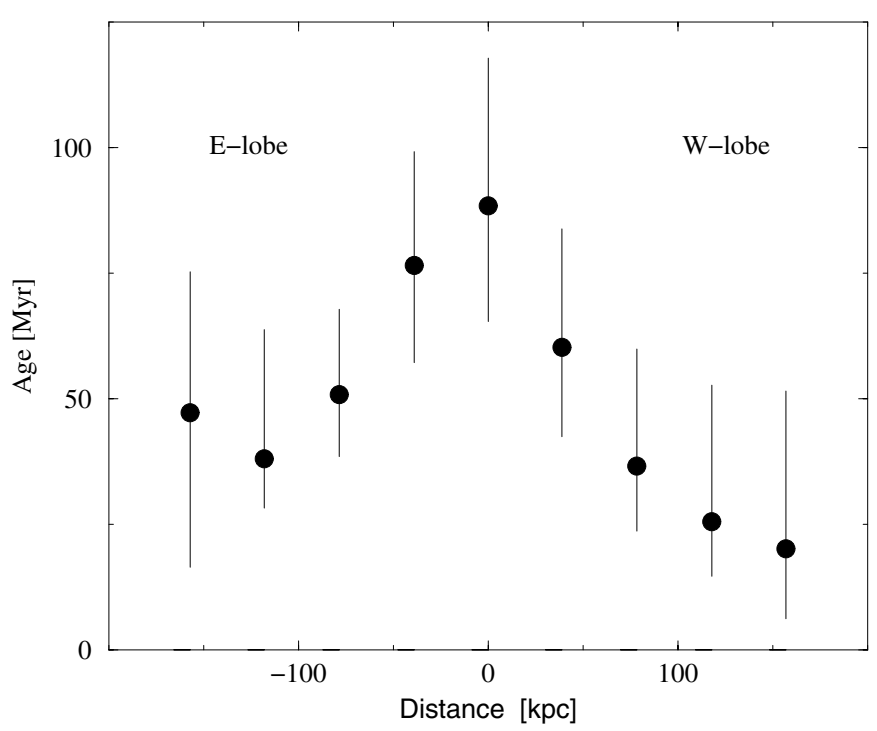

Fig. 8. Distribution of the synchrotron age of B2 0924+30 measured along its major axis which crosses the centre of the host galaxy IC 2476 .

spots in B2 0924+30 have certainly dispersed after exhaustion of the central source, so that their spectrum has been altered by expansion, as discussed by Carilli et al. (1991) for the Cyg A hot spots. In case of fully expanded former hot spots the initial injection spectrum, usually assumed to have $\alpha_{\mathrm{i}}=-0.5$, should have steepened to $\alpha=-1.0$, not very different from what is inferred from our fit to the total spectrum.

Using the images of B2 0924+30 at $\lambda \lambda 6.3,21,49$ and $92 \mathrm{~cm}$ we have determined the spectrum at different positions along the major axis of the source, measuring the flux density over selected regions with a beam equivalent area of $147^{\prime \prime}$ in order to ensure independent measurements. Using the SYNAGE software again and inferring a spectral index of $\alpha_{\mathrm{i}}=-1.0$, we have obtained the following results for the JP model: in the central part of the source the break frequency is around $2.5_{-1.0}^{+2.5} \mathrm{GHz}$, implying an age of $90_{-30}^{+30} \mathrm{Myrs}$, while away from the centre these values are $30_{-23}^{+67} \mathrm{GHz}$ and $26_{-11}^{+28}$ Myrs, respectively. These values are in rough agreement with what is derived from the integral radio spectrum. The results of the ageing analysis are shown in Fig. 8. The radiative age of two other relic radio galaxies i.e. B0917+75 ( 100 Myrs; Harris et al. 1993) and J1324-3138 (97 Myrs; Venturi et al. 1998) is comparable with the result we obtained for B2 0924+30. Alexander \& Leahy (1987) have found that the typical synchrotron lifetimes for low luminosity $3 \mathrm{CR}$ double radio galaxies are about 50 Myrs. Similar results (from 10 to 100 Myrs) for radiative ages were obtained by Parma et al. (1999) from the analysis of a sample of low luminosity B2 radio galaxies. Somewhat smaller ( $\$ 3$ Myrs) values of synchrotron lifetimes were found for a sample of 14 high luminosity $\left(\log L_{178 \mathrm{MHz}}>24 \mathrm{~W} \mathrm{~Hz}^{-1}\right)$ FRII sources (Liu et al. 1992).

On the whole, the source exhibits the spectral properties to be expected after exhaustion of the central AGN. The lateral spectral steepening seen in the lobes, and in particular the steepening toward the central region evident at high frequencies (Fig. 7) resembles the age gradient of the former highly relativistic particles. However, the spectral behaviour at the lowest frequencies (seen in Fig. 7c) is a surprise. Between 325 and $609 \mathrm{MHz}$ the central region is characterised by a very steep spectrum. The spectral index seen there in Fig. 7 is $\alpha_{325}^{609}=-1.8 \pm 0.1, \alpha_{609}^{1400}=-1.2 \pm 0.1, \alpha_{1400}^{4750}=-1.7 \pm 0.4$ at the lowest, intermediate and highest frequencies, respectively, of our study. We can only resort to speculation at this point. Figure 7c exhibits a misalignment of the low-frequency emission (having $\alpha_{325}^{609}=-1.8 \pm 0.1$ ) as compared to the overall major axis of B2 0924+30. Perhaps this emission reflects an even older period of activity, with jets pointing at a different direction, similar to what is inferred for "X-shaped" radio galaxies (Rottmann 2001).

The particle ages derived above are not very high. Since they reflect the lifetime of the relativistic particles after exhaustion of the central source, or after the termination of energisation of the former hot spots by the central engine, this means that once the energy transfer to the hot spots is stopped the whole radio source dies relatively quickly. The relatively short time scale resulting for the "switch-off" of B2 0924+30 naturally explains the relative paucity of such relic sources.

The particle propagation speeds implied by the above ages are of the order of $2000 \mathrm{~km} \mathrm{~s}^{-1}$ for particles having reached the outer lobe peripheries. These are some $112 \mathrm{kpc}$ (3.5) away from the presumed former hot spot locations in the two lobes.

Carilli et al. (1991) fitting KP and JP model spectra to the lobes of Cyg A obtained better fits of their data to the former model for the lobes of this radio galaxy. We have also applied a fit to the data with a KP model spectrum, anticipating that for an aged source like B2 $0924+30$ a pronounced difference in the goodness of the fit would result as compared to the JP model. Surprisingly, $\chi^{2}$ is about the same for both models, with about a factor of 2 difference in the resulting break frequencies.

\section{Summary and conclusions}

We have studied the radio spectrum of the relic source B2 0924+30. Using flux densities at 7 frequencies between 151 and $10550 \mathrm{MHz}$ we have estimated the mean age of the relativistic particles responsible for the steep spectrum of the synchrotron emission of the source. This was accomplished by fitting a model spectrum to the data, allowing for synchrotron and inverse Compton losses of particles with an isotropic pitch angle distribution as worked out by Jaffe \& Perola (1973). The average particle age is $54_{-11}^{+12}$ Myrs, and the low-frequency spectral index is determined as $\alpha_{\mathrm{i}}=-0.87 \pm 0.09$, probably reflecting the spectrum of an initially injected electron population which has undergone synchrotron losses.

Images at $\lambda \lambda 6.3,21,49$ and $92 \mathrm{~cm}$ have been subjected to a study of the spectrum and particle ages across the diffuse radio lobes. The spectrum between the four wavelengths is generally rather steep. At high frequencies $\alpha_{1400}^{4750}=-1.4 \pm 0.1$ along the lobes' ridges, and $\alpha_{1400}^{4750}=-1.7 \pm 0.4$ at the position of the host galaxy IC 2476. Away from the lobe ridges, the spectrum steepens further, assuming mean values of $\alpha_{1400}^{4750}=$ $-1.7 \pm 0.2$. An unusual spectral behaviour is seen in the central region of B2 $0924+30$. The spectrum being extremely steep at the lowest frequencies $\left(\alpha_{325}^{609}=-1.8 \pm 0.1\right)$ first flattens to 
$\alpha_{609}^{1400}=-1.2 \pm 0.1$ at intermediate ones, while steepening to $\alpha_{1400}^{4750}=-1.7 \pm 0.4$ again at the highest frequencies of our study. We hypothesise that this structure, which also exhibits a misalignment with the overall major axis of B2 $0924+30$, is a relic of a still older period of activity.

The break frequencies (particle ages) vary between $v_{\mathrm{b}}=$ $30_{-23}^{+67} \mathrm{GHz}\left(26_{-11}^{+28} \mathrm{Myrs}\right)$ along the lobes' ridges and $v_{\mathrm{b}}=$ $2.5_{-1.0}^{+2.5} \mathrm{GHz}\left(90_{-30}^{+30} \mathrm{Myrs}\right)$ in the central part of the relic galaxy. The corresponding particle advance speed is of the order of $2000 \mathrm{~km} \mathrm{~s}^{-1}$. The radiative age of B2 $0924+30$ is comparable with the age of other relic radio galaxies (e.g. B0917+75 and J1324-3138) and with low luminosity radio galaxies from the $3 \mathrm{CR}$ and $\mathrm{B} 2$ samples.

With the relatively low particle age since "switch-off" inferred from our study of this genuine relic source, aged radio sources would easily escape detection in the GHz-frequency range. It is therefore important to search for such objects in low-frequency radio continuum surveys such as the VLA LowFrequency Sky Survey (VLSS, Kassim et al. 2003). In the not too distant future, the Low Frequency Array (LOFAR, Röttgering 2003) will provide the tool to search for exhausted radio galaxies and quasars. Their investigation and census will fill a gap in the picture of AGN evolution and bears significant cosmological importance.

Acknowledgements. M.J. acknowledges the Deutsche Forschungsgemeinschaft for the award of a postdoctoral fellowship (GRK 787). KHM was supported by a Marie-Curie-Fellowship of the European Commission. We thank the anonymous referee for comments and suggestions. This research is partly based on observations performed with the 100-m telescope of the MPIfR (Max-Planck-Institut für Radioastronomie) at Effelsberg.

\section{References}

Alexander, P., \& Leahy, J. P. 1987, MNRAS, 225, 1

Arp, H. 1977, in Décalages vers le Rouge et Expansion de L'Univers, ed. C. Balkowski, \& B. E. Westerlund, Proc. IAU Coll., 37, 377

Baars, J. W. M., Genzel, R., Pauliny-Toth, I. I., \& Witzel, A. 1977, A\&A, 61, 99

Beckeer, R., White, R., \& Helfand, D. 1995, ApJ, 450, 559

Canosa, C. M., Worrall, D. M., Hardcastle, M. J., \& Birkinshaw, M. 1999, MNRAS, 310, 30

Carilli, C. L., Perley, R. A., Dreher, J. W., \& Leahy, J. P. 1991, ApJ, 383,554

Colla, G., Fanti, C., Ficarra, A., et al. 1970, A\&AS, 1, 281

Colla, G., Fanti, C., Fanti, R., et al. 1975, A\&AS, 20, 1
Condon, J. J., Cotton, W. D., Greisen, E. W., et al. 1998, AJ, 115, 1693

Cordey, R. A. 1987, MNRAS, 227, 695

Ekers, R. D., Fanti, R., Lari, C., \& Ulrich, M.-H. 1975, Nature, 258, 584

Ekers, R. D., Fanti, R., Lari, C., \& Parma, P. 1981, A\&A, 101, 194

Emerson, D. T., Klein, U., \& Haslam, C. G. T. 1979, A\&A, 76, 92

Falco, E. E., Kurtz, M. J., Geller, M. J., et al. 1999, PASP, 111, 438

Fanaroff, B. L., \& Riley, J. M. 1974, MNRAS, 167, 31

Gioia, I. M., Gregorini, L., \& Klein, U. 1982, A\&A, 116, 164

Giovannini, G., Feretti, L., Gregorini, L., \& Parma, P. 1988, A\&A, 199, 73

Goldshmidt, O., \& Raphaeli, Y. 1994, ApJ, 431, 586

Gregorini, L., Klein, U., Parma, P., Schlickeiser, R., \& Wielebinski, R. 1992, A\&AS, 94, 13

Harris, D. E., Stern, C. P., Willis, A. G., \& Dewdney, P. E. 1993, AJ, 105,769

Jaffe, W. J., \& Perola, G. C. 1973, A\&A, 26, 423

Kaiser, C. R., \& Cotter, G. 2002, MNRAS, 336, 649

Kardashev, N. S. 1962, SvA, 6, 317

Kassim, N. E., Lane, W. M., Cohen, A. S., et al. 2003, A\&AS, 203, 57.09

Kempner, J. C., Blanton, E. L., Clarke, T. E., et al. 2003, in Proc. of The Riddle of Cooling Flows in Galaxies and Clusters of Galaxies, ed. T. Reiprich, J. Kempner, \& N. Soker [arXiv: astro-ph/0310263]

Klein, U., Mack, K.-H., Gregorini, L., \& Parma, P. 1995, A\&A, 303, 427

Komissarov, S. S., \& Gubanov, A. G. 1994, A\&A, 285, 27

Liu, R., Pooley, G., \& Riley, J. M. 1992, MNRAS, 257, 545

Mahdavi, A., Geller, M. J., Böhringer, H., Kurtz, M. J., \& Ramella, M. 1999, ApJ, 518, 69

Miley, G. 1980, ARA\&A, 18, 165

Murgia, M. 1996, Laurea Thesis, University of Bologna

Nilson, P. 1973, Uppsala General Catalogue of Galaxies, Acta Universitatis Upsaliensis, Nova Acta Regiae Societatis Scientarum Upsaliensis, SER. V:A., 1

Pacholczyk, A. G. 1970, Radio Astrophysics (San Francisco: Freeman)

Parma, P., Murgia, M., Morganti, R., et al. 1999, A\&A, 344, 7

Parma, P., de Ruiter, H. R., Murgia, M., \& Mack, K.-H. 2003, in The Cosmic Cauldron, 25th Meet. of the IAU, Joint Discussion, 10, 18

Rengelink, R. B., Tang, Y., de Bruyn, A. G., et al. 1997, A\&AS, 124, 259

Röttgering, H. 2003, NewAR, 47, 405

Rottmann, H. 2001, Ph.D. Thesis ${ }^{2}$, University of Bonn

Schlickeiser, R., Sievers, A., \& Thiemann, H. 1987, A\&A, 182, 21

Sieber, W., Haslam, C. G. T., \& Salter, C. J. 1979, A\&A, 74, 361

Venturi, T., Bardelli, S., Morganti, R., \& Hunstead, R. W. 1998, MNRAS, 298, 1113

White, R. A., Bliton, M., Bhavsar, S. P., et al. 1999, AJ, 118, 2014

\footnotetext{
${ }^{2}$ PDF file available on the web: http://hss.ulb.uni-bonn.de/diss_online/ math_nat_fak/2001/rottmann_helge/index.htm
} 